\title{
The distal fascicle of the anterior inferior tibiofibular ligament as a cause of tibiotalar impingement syndrome: a current concepts review
}

\author{
Michel P. J. van den Bekerom · Eric E. J. Raven
}

Received: 12 August 2006/ Accepted: 11 December 2006/Published online: 20 January 2007

(C) Springer-Verlag 2007

\begin{abstract}
Impingement syndromes of the ankle involve either osseous or soft tissue impingement and can be anterior, anterolateral, or posterior. Ankle impingement syndromes are painful conditions caused by the friction of joint tissues, which are both the cause and the effect of altered joint biomechanics. The distal fascicle of the anterior inferior tibiofibular ligament (AITFL) is possible cause of anterior impingement. The objective of this article was to review the literature concerning the anatomy, pathogenesis, symptoms and treatment of the AITFL impingement and finally to formulate treatment recommendations. The AITFL starts from the distal tibia, $5 \mathrm{~mm}$ in average above the articular surface, and descends obliquely between the adjacent margins of the tibia and fibula, anterior to the syndesmosis to the anterior aspect of the lateral malleolus. The incidence of the accessory fascicle differs very widely in the several studies. The presence of the distal fascicle of the AITFL and also the contact with the anterolateral talus is probably a normal finding. It may become pathological, due to anatomical variations and/or anterolateral instability of the ankle resulting from an anterior talofibular ligament injury. When observed during an ankle arthroscopy, the surgeon should look for the criteria described to decide whether it is pathological and considering resection
\end{abstract}

M. P. J. van den Bekerom - E. E. J. Raven

Department of Orthopaedic Surgery, Gelre Hospitals,

Apeldoorn, The Netherlands

M. P. J. van den Bekerom ( $₫)$

Department of Surgery and Traumatology,

Gelre Hospitals, Location Lukas, P.O. Box 9014,

7300 Apeldoorn, The Netherlands

e-mail: Bekerom@gmail.com of the distal fascicle. The presence of the AITFL and the contact with the talus is a normal finding. An impingement of the AITFL can result from an anatomical variant or anteroposterior instability of the ankle. The diagnosis of ligamentous impingement in the anterior aspect of the ankle should be considered in patients who have chronic ankle pain in the anterolateral aspect of the ankle after an inversion injury and have a stable ankle, normal plain radiographs, and isolated point tenderness on the anterolateral aspect of the talar dome and in the anteroinferior tibiofibular ligament. The impingement syndrome can be treated arthroscopically.

Keywords Ankle - Impingement syndrome · Anterior inferior tibiofibular ligament .

Accessory fascicle

\section{Introduction}

Impingement syndromes of the ankle involve either osseous or soft tissue impingement and can be anterior, anterolateral, or posterior [5, 25]. Ankle impingement syndromes are painful conditions caused by the friction of joint tissues, which are both the cause and the effect of altered joint biomechanics [14]. The leading causes of impingement lesions are post-traumatic ankle injuries, usually ankle sprains, resulting in chronic ankle pain [33]. According to the ISAKOS definition, anterior ankle impingement is a pain syndrome characterised by anterior ankle pain on activity. Recurrent dorsiflexion is often the cause. On investigation, there is pain on palpation at the antero/medial and/or anterolateral aspect of the ankle joint. Some swelling and/ 
or limitations of dorsiflexion are present [32]. The first reported impingement syndromes about the ankle have evolved osseous impingement and generally have been noted in athletes whose sports necessitated sudden acceleration, jumping, and extremes of dorsiflexion or plantar flexion. Impingement of the ankle was first described in the English language literature by Morris [20], in 1943, in five patients who had what he called the athlete's ankle. This was later called footballer's ankle in the report of McMurray [18].

Soft tissue impingement of the ankle is a common cause of chronic ankle pain that usually arises at the lateral and/or anterolateral compartment of the ankle joint following an inversion injury [8]. It is estimated that approximately $3 \%$ of ankle sprains may lead to anterolateral impingement [32]. Three types of intraarticular soft tissue lesions that lead to these complaints have been described: the meniscoid lesion, synovitis, and the distal fascicle of the anterior inferior tibiofibular ligament (AITFL) [4, 6, 8, 9, 12, 16, 19, 28]. The formation of tibiotalar spurs due to repetitive capsule traction therefore does not seem plausible reason for anterior ankle impingement [29].

Impinging soft tissue pathology in the ankle was first described by Wolin et al. as a "meniscoid mass or lesion" [34]. They described the lesion as a membrane shaped hyalinised scar tissue obstructing the lateral talofibular articulation in nine patients with persistent symptoms over the anterolateral corner of the ankle after an inversion trauma [34]. Some authors thought this lesion was formed from the torn ends of the anterior tibiofibular ligament $[3,27]$. However there was no ligamentous tissue found on histologic examination of this lesion [9]. Guhl thought the lesion was of synovial origin [11]. Irritation of the joint by bleeding and a torn capsule or a torn anterior tibiofibular ligament are thought to be the underlying factors for developing post-traumatic synovitis in the ankle [17] which in unstable ankles may lead to formation of fibrosis and granulation tissue. Although synovitis and the meniscoid lesion have been referred frequently, impingement by a separated fascicle of the AITFL is a relatively new and unknown pathology. Bassett et al. [4] were the first who reported a ligamentous etiology of impingement of the anterior aspect of the ankle. The presence of the so-called accessory AITFL, as described by Nikolopoulos was confirmed by them [21]. However they called this accessory ligament, the distal fascicle of the normal AITFL [4]. The distal part of the fascicle rubs against the talus, and that this causes pain at the ankle [1, 2, 4, 8, 12, 22].

The objective of this article was to review the literature concerning the anatomy, pathogenesis, symptoms and treatment of the AITFL impingement and finally to formulate treatment recommendations.

\section{Anatomy of the anterior inferior tibiofibular ligament}

The distal tibiofibular joint was classically described as a syndesmosis between the rough, triangular surface on the medial and distal aspect of the fibular shaft and the fibular notch of the distal end of the tibia. The tibiofibular syndesmosis is established by three ligaments: the interosseous ligament, the posterior inferior tibiofibular ligament, and the AITFL [7, 10, 15, 24, 26].

The AITFL is a flat band becoming thicker from superior to inferior [24]. The ligament starts from the distal tibia, $5 \mathrm{~mm}$ in average above the articular surface, and descends obliquely between the adjacent margins of the tibia and fibula, anterior to the syndesmosis to the anterior aspect of the lateral malleolus [7, 22]. Its length ranges from 12 to $20 \mathrm{~mm}$ [4, 22]; its thickness, from 1 to $3 \mathrm{~mm}[4,22]$; and its width ranges from 7 to $12 \mathrm{~mm}$ at the fibular insertion and 9 to $22 \mathrm{~mm}$ at the tibial insertion [22]. This ligament is the weakest of the syndesmotic ligaments and is the first to yield to forces that promote an external rotation of the fibula around its longitudinal axis [15]. Resection of the accessory ligament did not disturb the stability of the syndesmosis, a finding that was in accordance with the results reported by Rasmussen et al. which suggests that mobility is minimally influenced by isolated cutting of the AITFL [23].

Nikolopoulos et al. studied 24 cadaveric ankles to describe the anatomy of the AITFL [22]. In five ankles the AITFL appeared to consist of two layers, one superficial and one deep, which were separated by a thin fibrofatty septum [22, 24]. However, the total thickness of these two layers did not exceed the average thickness of the AITFL in the rest of the specimens [22]. In five ankles, the articulated surfaces of the distal tibia and fibula that formed the syndesmosis were covered by articular cartilage and were surrounded by synovial bursa [22]. A completely separate accessory anteroinferior tibiofibular ligament was present in 22 ankles (Fig. 1). Nikolopoulos was the first to describe this accessory ligament [21]. However the results of his study were never published in English literature [26]. Nikolopoulos believed that this anatomical structure represents a separate ligament rather than a distal component of the AITFL [21]. The fact that there was clearly a fibrofatty septum that separates the AITFL from the accessory fascicle, similar to the one that covered the intermediate space between the interosseous and the anterior or the interosseous and the 


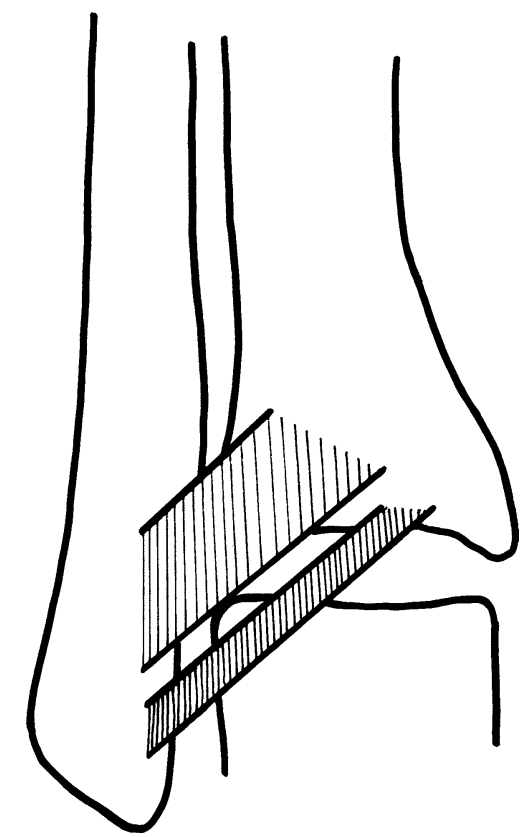

Fig. 1 The distal fascicle in relation to the anterior inferior tibiofibular ligament

posterior tibiofibular ligaments, suggested that this fascicle could probably be considered an independent accessory ligament [22]. Some other authors described this accessory ligament as a complete separate distal fascicle of the AITFL $[4,15,26]$.

Ray and Kriz [24] founded in 10 of the 46 specimens an accessory ligament. This ligament was parallel and distal to the AITFL. The fibres of the accessory ligament descended obliquely from the lateral, anterior, and distal borders of the tibia to the anteromedial aspect of the lateral malleolus, approximating the fibular insertion of the anterior talofibular ligament (ATFL).

Its length ranges from 17 to $22 \mathrm{~mm}$, its thickness from 1 to $2 \mathrm{~mm}$, and its width from 3 to $5 \mathrm{~mm}$. The maximum, minimum, mean, and standard deviation for the length, width, gap, and angle of declination for the inferior fibre bundle are provided in Table 1. The ligament was not covered by synovial tissue, ran deeper than the AITFL and was described as being intraarticular, although how this was determined was not stated by the authors. The accessory ligament crossed

Table 1 Characteristics of the inferior fascicle of the AITFL [24]

\begin{tabular}{lccr}
\hline & Maximum & Minimum & \multicolumn{1}{c}{ Mean } \\
\hline Ligament length $(\mathrm{cm})$ & 3.4 & 2.1 & $2.6 \pm 0.3$ \\
Ligament width $(\mathrm{cm})$ & 1.3 & 0.2 & $0.4 \pm 0.2$ \\
Gap (cm) & 0.5 & 0.1 & $0.2 \pm 0.1$ \\
Declination angle (deg) & 155.0 & 121.5 & $136.3 \pm 7.4$ \\
\hline
\end{tabular}

the superior lateral margin of the ankle joint. The accessory ligament was separated from the AITFL by a distinct fibrofatty septum. The ligament could even be divided in two, three, or more fascicles, increasing in length from superior to inferior [24].

The incidence of the accessory fascicle differs very widely in the several studies (21-92\%) [2, 4, 22, 24]. The discrepancies in the incidence between the several studies were probably due to the different definitions of a separate fascicle. Ray and Kriz did not consider it a separate fascicle if any digitations were present between the fascicle and the main body of the AITFL [24]. The incidence was up to $92 \%$, which implied that the ligament may be considered as a normal finding. Vessels from the anterior peroneal artery penetrate through the interlaminar spaces [26]. It is not uncommon to have branches from the anterior tibial artery or peroneal artery or a branch arising from the anastomosis between these two arteries pass between these fascicles [11]. The most inferior fibres of the ligament merged at their fibular attachment with the origin of the ATFL [24].

Ray and Kriz [24] studied 23 cadaveric ankles. A classification scheme was designed to categorise the anatomical variations observed in the AITFL (Table 2).

\section{Pathomechanism of the impingement syndrome}

It should be stressed that most studies were carried out in vitro and do not take muscular tonus and the effects of weightbearing into consideration, we performed a review of the pathomechanism of the impingement syndrome caused by the distal fascicle of the AITFL.

Bassett et al. correlated the presence of this accessory ligament with pain from impingement in the anterolateral aspect of the ankle in patients with a history of inversion sprains [4]. They postulated that the posttraumatic anterolateral hyperlaxity, due to an injured ATFL, resulted in anterior extrusion of the talar dome with dorsiflexion, which now contacted the inferior fascicle of the AITFL with more pressure and friction [4]. This in turn might set up an environment favouring ligamentous impingement of the anterior aspect of the ankle [4].

Akseki et al. stated that the variations in width, length, and obliquity of the fascicle may be related to the pathological behaviour of the fascicle [2]. Mean width and length of the fascicle with bending during dorsiflexion and dorsiflexion-eversion were significantly higher than the fascicle without bending. Therefore this wider and longer fascicles had more potential to 
Table 2 AITFL classification system [24]

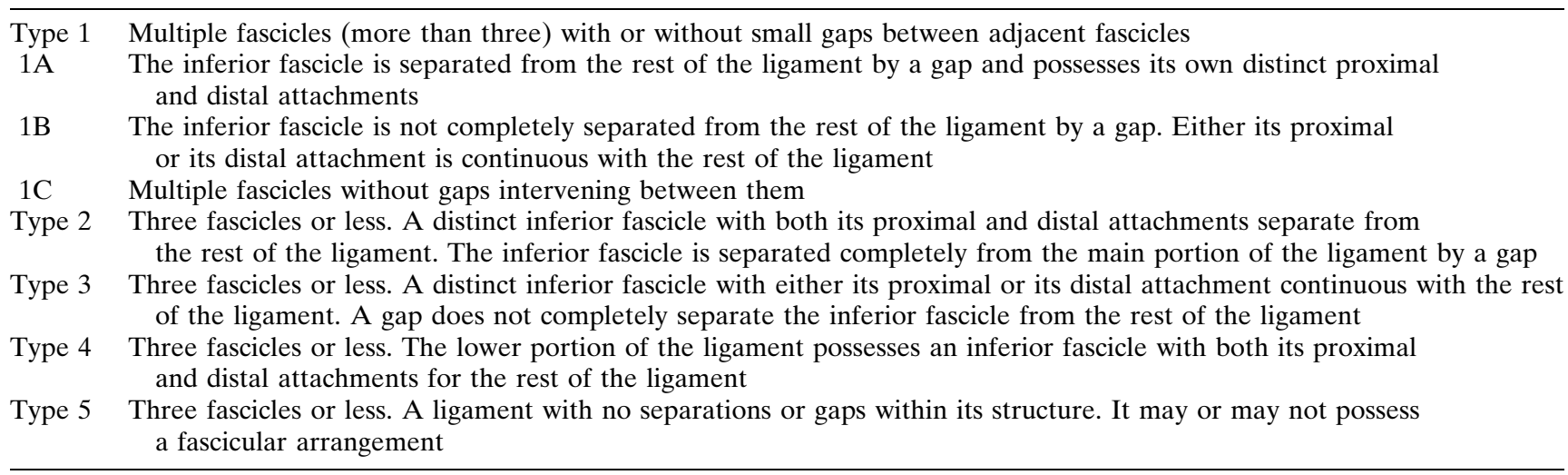

become pathological than thinner ones. They also conclude that if the fibular insertion point is far from the joint level, the fascicle has more potential to become pathological. These findings might be useful during arthroscopic procedures of the ankle.

During dorsiflexion of the ankle the posterior shift of the anterior talus below the tibial plafond in ATFL intact cases was observed. Following incision of the ATFL this posterior shift of the talus was not observed; the talus remained in an anterior position and thus in contact with the fascicle [2]. Lesion of the ATFL led to bending of the fascicle with dorsiflexion and dorsiflexion-inversion in the specimens which the bending sign was negative. Lesion of the ATFL led also to contact between the talus and the fascicle in the ankles which showed no contact previously [2]. After sectioning the ATFL the anteroposterior laxity of the ankle increases especially in dorsiflexion [4, 13].

In $89.3 \%$ of the cases contact was observed between the ATFL and the anterolateral corner of the talus [2]. This contact may be normal in some of the cases. Contact was observed at an average of $12^{\circ}$ of dorsiflexion of the ankle [4]. Bending of the accessory ligament, indicating further tensioning of its ligamentous bands caused by impingement of the talus, this was noted in an extent of dorsiflexion that varied from $5^{\circ}$ to $10^{\circ}$ (average $7^{\circ}$ ) [22]. Maximum dorsiflexion of the foot relieved the contact between the ligament and the superolateral corner of the talus [22]. In other studies the contact was also observed in neutral position [2, 24].

In one study the contact between the fascicle and talar dome was observed throughout the whole range of motion of the ankle [1]. They called this the "arthroscopic impingement test," and it was positive in all of the cases with AITFL impingement they have studied [1]. Contact between the accessory fascicle and the talus was also observed during eversion of the foot, being more prominent when the foot was placed in a dorsiflexion-eversion position [22]. No interaction of the accessory ligament with the talus was noted during ankle plantar flexion [22].

In $82.9 \%$ of the cases with AITFL impingement Ray and Kriz [24] found an anterior abraded articular region of the talus. Akseki et al. [2] found this in $17 \%$ of the cases. In the study of Basset et al. [4] 71\% of the patients, had an area of abraded articular cartilage on the anterolateral surface of the talar dome. There is no clarity if this abraded cartilage region is an anatomical variant of the talus or a pathological remodelling of the bone due to true ligamentous impingement [4].

On base of these studies we conclude that anterolateral hyperlaxity results in anterior extrusion of the talar dome with dorsiflexion, which contacts the inferior fascicle of the AITFL with more pressure and friction. This hyperlaxity was most likely due to an injured ATFL. Wider and longer fascicles and a fibular insertion point far from the joint level have more potential to become pathological. Often contact between the AITFL and the superolateral corner of the talus and an abraded region of the cartilage of the talus can be observed during arthroscopy.

\section{Diagnosis}

The diagnosis of ligamentous impingement in the anterior aspect of the ankle should be considered in patients who have chronic ankle pain in the anterolateral aspect of the ankle after an inversion sprain and who have a stable ankle, normal plain radiographs, and isolated point tenderness on the anterolateral aspect of the talar dome and in the anteroinferior tibiofibular ligament [4]. A popping sensation and aggravation of pain with dorsiflexion and eversion were noted [2, 4]. This audible popping is usually not seen in other causes of impingement of the anterior aspect of the ankle [4]. 
Fig. 2 There is contact between the distal fascicle of AITFL and the talus in dorsiflexion with bending of this fascicle
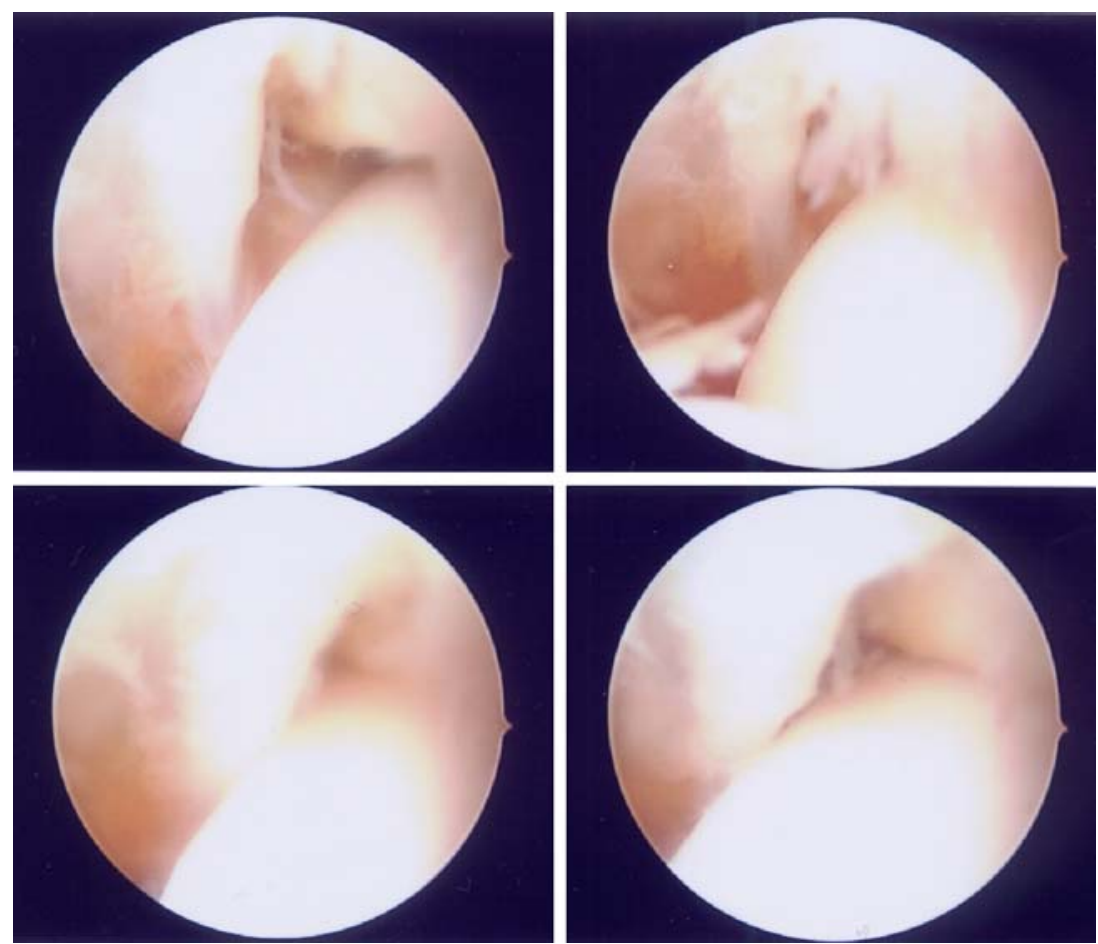

Pain in the beginning of plantarflexion-inversion may alert the physician for an impinging AITFL [2].

It is usually impossible to distinguish the source of pain in patients with both instability and impingement. So if the patient complains of giving way and frequent sprains, the surgeon may choose a reconstructive procedure [1]. But it should be noted that a painful lesion in the joint (synovitis, meniscoid lesion, or an impinging fascicle of the AITFL) may cause reflex sprains [1]. This process may confuse decision making in this type of combined pathology. The differential diagnosis for pain in the lateral aspect of the ankle after an inversion sprain includes osteochondral fracture, instability of the lateral aspect of the ankle, synovitis or rupture of the peroneal tendon, and ligamentous impingement in the anterior aspect of the ankle, with the latter being the least common [4].

\section{Treatment}

Patients should be treated conservatively after an inversion sprain of the ankle for at least 6 months before operative intervention is considered [5]. This is with the exception of patients with an osteochondral fracture. Akseki et al. observed that conservative management with physical therapy, NSAIDs and bracing in patients with instability symptoms, for a period of 3 months, failed in all 21 patients [1].
Not every patient with chronic ankle instability needs a reconstructive procedure [1]. If the history, physical examination, and other diagnostic tests suggest impingement in addition to instability, arthroscopic debridement of the soft tissue lesion may be all that is needed [1. In cases with an area of abraded articular cartilage on the anterolateral surface of the talar dome, further debridement of this region was required [4]. At long-term follow-up arthroscopic excision of soft tissue overgrowth and osteophytes proved to be an effective way of treating anterior impingement of the ankle in patients who had no narrowing of the joint space [30]. Patients with less than 2 years of ankle pain before surgery for anterior ankle impingement showed significant better scores in pain, swelling, ability to work, and engagement in sports [31].

The decision to perform an arthroscopy of the ankle is typically based on the patient's history and physical examination $[9,19,28]$. Arthroscopically resection of the distal fascicle of the AITFL should be considered when there (Fig. 2): (1) is contact between the AITFL and the talus was prominent also in the beginning of plantarflexion-inversion of the ankle, (2) is increased contact between the talus and the ligament and this continued until maximum dorsiflexion with abrasion of the articular cartilage (the surgeon should remember that this finding may be normal), (3) bending of the fascicle on the anterolateral edge of the talus with 
dorsiflexion and dorsiflexion-inversion, (4) is a distally inserting fascicle on the fibula, close to the origin of the ATFL on the fibula. This finding may be missed if the distraction is preserved throughout the procedure $[2,24]$. For this reason, temporarily relieving distraction is advised, when a distal fascicle is seen during arthroscopy [2, 22].

Only three studies reported on the results of the treatment of AITFL impingement [1, 4, 12]. Akseki et al. [1] arthroscopically resected the distal fascicle of the AITFL in 21 patients with chronic ankle pain after an ankle sprain. During the procedure an impinging distal fascicle of the AITFL was found in all cases. Following anterolateral synovectomie, the fascicle was excised. At an average follow-up of 3 years good to excellent results were obtained in 17 patients. Nineteen patients were satisfied with the procedure and 17 patients returned to previous level of activity. Two patients who had mild laxity were graded as poor because neuromas of the terminal branches of the superficial peroneal nerve. These patients became asymptomatic after an injection of steroids.

Bassett et al. [4] treated seven patients who had talar impingement by an AITFL and they were followed for a mean of 39 months. Two patients had arthroscopy and five had an arthrotomy. In all patients, a thickened distal fascicle of the AITFL was resected. Five patients also needed debridement of an area of abraded hyaline cartilage on the anterolateral aspect of the talar dome at the point where the distal fascicle made contact. Post-operative management consisted of 2-4 days of immobilisation, followed by progressive weightbearing. Four patients reported no pain in their ankle or limitation in activity, and the results were considered to be excellent. Two patients reported improvement, with only occasional pain related to overuse, and the result was considered good. The last patient had been followed for only 6 months with excellent results.

Horner and Liu [12] treated nine athletes with anterolateral ankle pain during and following athletic activities without history of ankle injury. After 3 months conservative management each patient was explored arthroscopically. Patients' outcomes were rated subjectively and objectively based on the UCLA ankle scoring system. Pathological characteristics were easily identifiable through arthroscopy. The structure may be resected during this procedure without sacrificing the stability of the ankle joint. They report that good to excellent results can be expected from this procedure including in the highest level of athleticism at an average follow-up of 18 months.
The decision to perform an arthroscopy of the ankle is typically based on the patient's history and physical examination. When an impinging distal fascicle of the AITFL and an abraded anterolateral region of the talus were observed during an ankle arthroscopy, the surgeon should look for the criteria described above to decide whether it is pathological and needs to be resected.

\section{Conclusion}

Because of the lack of evidence only preliminary conclusions can be drawn. The AITFL started from the distal tibia, $5 \mathrm{~mm}$ in average above the articular surface, and descended obliquely between the adjacent margins of the tibia and fibula, anterior to the syndesmosis to the anterior aspect of the lateral malleolus. The incidence of the accessory fascicle differs very widely in the several studies. The presence of the distal fascicle of the AITFL and also the contact with the anterolateral talus is probably a normal finding. It may become pathological, however, due to anatomical variations and/or anterolateral instability of the ankle resulting from an ATFL injury. When observed during an ankle arthroscopy, the surgeon should look for the criteria described to decide whether it is pathological and considering resection of the distal fascicle.

\section{References}

1. Akseki D, Pinar H, Bozkurt M, et al (1999) The distal fascicle of the anterior inferior tibiofibular ligament as a cause of anterolateral ankle impingement. Acta Orthop Scand 70(5):478-482

2. Akseki D, Pinar H, Yaldiz K, et al (2002) The anterior tibiofibular ligament and talar impingement: a cadaveric study. Knee Surg Sports Traumatol Arthrosc 10:321-326

3. Andrews JR, Drez DJ, McGinthy JB (1984) Symposium: arthroscopy of joints other than the knee. Contemp Orthop 9:71-100

4. Bassett FH III, Gates HS III, Billys JB, et al (1990) Talar impingement by the anteroinferior tibiofibular ligament. J Bone Joint Surg 72A:55-59

5. Canale ST (1998) Ankle injuries. In: Canale ST (ed) Cambell's operative orthopaedics, 9th edn. CV Mosby, St Louis, pp 1079-1112

6. DeBerdino MT, Arciero RA, Tayler CD (1997) Arthroscopic treatment of soft-tissue impingement of the ankle in athletes. Arthroscopy 13(4):492-498

7. Draves DJ (1986) Osteology and arthrology of the knee and leg. In: Draves DJ (ed) Anatomy of the lower extremity. Williams \& Wilkins, Baltimore, p 95

8. Ferkel RD (1994) Differential diagnosis of chronic ankle sprain pain in the athlete. Sports Med Arthrosc Rev 2:274273 
9. Ferkel RD, Karzel RP, Pizzo WD, et al (1991) Arthroscopic treatment of antero-lateral impingement of the ankle. Am J Sports Med 19(5):440-446

10. Gray H (1973) Articulations of the lower limb. In: Goss CM (ed) Gray's anatomy, 29th edn. Lea \& Febiger, Philadelphia

11. Guhl J (1993) Foot and ankle arthroscopy, vol 1, 2nd edn. Slack, Thorofare, pp 88-105

12. Horner G, Liu S (1996) Arthroscopic treatment of talar impingement by the accessory anteroinferior tibiofibular ligament. Arthroscopy 12:384-385

13. Johnson EE, Markolf KL (1983) The contribution of the anterior talofibular ligament to ankle laxity. J Bone Joint Surg 65:81-88

14. Kleiger B (1982) Anterior tibiotalar impingement syndromes in dancers. Foot Ankle 3:69-73

15. Kelikian H, Kelikian AS (1985) Correlative anatomy of the ankle joint. In: Kelikian H, Kelikian AS (eds) Disorders of the ankle. WB Saunders, Philadelphia, p 1

16. Liu HS, Raskin A, Osti L, et al (1994) Arthroscopic treatment of anterolateral ankle impingement. Arthroscopy 10(2):215-218

17. Lundeen RO (1990) Arthroscopic evaluation of the traumatic injuries to the ankle and foot. Part: II chronic posttraumatic pain. J Foot Surg 29(1):59-71

18. McMurray TP (1950) Footballer's ankle. J Bone Joint Surg 32B(1):68-69

19. Meislin JR, Rose JD, Parisien SJ, et al (1993) Arthroscopic treatment of synovial impingement of the ankle. Am J Sports Med 21(2):186-189

20. Morris LH (1943) Athlete's ankle. J Bone Joint Surg 25:220

21. Nikolopoulos CE (1982) Anterolateral instability of the ankle joint: an anatomical, experimental and clinical study. Thesis, University of Athens, Athens, Greece

22. Nikolopoulos CE, Tsirikos AI, Sourmelis S, et al (2004) The accessory anteroinferior tibiofibular ligament as a cause of talar impingement. A cadaveric study. Am J Sports Med 32(2):389-395
23. Rasmussen O, Tovborg-Jensen I, Boe S, et al (1982) Distal tibiofibular ligaments: analysis of function. Acta Orthop Scand 53:681-686

24. Ray RG, Kriz BM (1991) Anterior inferior tibiofibular ligament. Variations and relationship to the talus. J Am Podiatr Med Assoc 81:479-485

25. Renstrom PAFH (1994) Persistently painful sprained ankle. J Am Acad Orthop Surg 2:270-280

26. Sarrafian SK (1983) Anatomy of the foot and ankle. Descriptive, topographic, functional. J.B. Lippincott, Philadelphia

27. Schonholtz GJ (1986) Arthroscopic surgery of the shoulder, elbow and ankle. Charles C Thomas, Springfield, pp 69-71

28. Thein R, Eichenblat M (1992) Arthroscopic treatment of sports related synovitis of the ankle. Am J Sports Med 20(5):496-498

29. Tol JL, van Dijk CN (2004) Etiology of the anterior ankle impingement syndrome: a descriptive anatomical study. Foot Ankle Int 25(6):382-386

30. Tol JL, Verheyen CP, van Dijk CN (2001) Arthroscopic treatment of anterior impingement in the ankle. J Bone Joint Surg Br 83(1):9-13

31. van Dijk CN, Tol JL, Verheyen CC (1997) A prospective study of prognostic factors concerning the outcome of arthroscopic surgery for anterior ankle impingement. Am J Sports Med 26(6):737-745

32. van Dijk CN (2005) Ankle impingement. In: Chan KM, Karlsson J (eds) ISAKOS/FIMS World consensus conference on ankle instability, Hong Kong

33. Umans H (2002) Ankle impingement syndromes. Semin Musculoskelet Radiol 6:133-139

34. Wolin I, Glassman F, Sideman S, et al (1950) Internal derangement of the talofibular component of the ankle. Surg Gynecol Obstet 91:193-200 